\title{
A PROOF AND EXTENSION OF DEHN'S LEMMA
}

\author{
BY ARNOLD SHAPIRO AND J. H. C. WHITEHEAD
}

Communicated by Norman Steenrod, April 25, 1958

1. Introduction. C. D. Papakyriakopoulos [2] has recently proved Dehn's lemma [1]. His proof has the merit that the basic construction (the tower) and the crucial lemmas apply to the sphere theorem as well as Dehn's lemma. However, if one is content with Dehn's lemma the proof can be simplified. In this note we first give a simplified proof of Dehn's lemma and then prove an analogous theorem for surfaces with more than one boundary curve.

By a surface of type $(p, r)$ we mean a connected, compact, orientable surface of genus $p$ with a boundary consisting of $r 1$-spheres. Thus the Euler characteristic of such a surface is $2(1-p)-r$. A surface of type $(0,1)$ is called a disc. A Dehn surface of type $(p, r)$ is a polyhedral singular surface of type $(p, r)$ with no singularities on the boundary. Our extension of Dehn's lemma refers to surfaces of type $(0, r)$. In order to state it we need two more definitions.

Let $M$ be a connected 3-manifold and let $\tilde{M}$ be a universal cover of $M$. The manifold $\widetilde{M}$ is orientable and the set of elements of $\pi_{1}(M)$ $=\pi_{1}\left(M, x_{0}\right)$ which correspond, in the usual way, to orientationpreserving covering transformations of $\tilde{M}$ is a sub-group of index 2 . We denote it by $\omega(M)$. Thus $M$ is orientable if, and only if, $\omega(M)$ $=\pi_{1}(M)$.

Let $C_{1}, \cdots, C_{r}$ be nonsingular closed curves in $M$ and let each $C_{r}$ be oriented and joined to $x_{0}$ by a path in $M$ so as to represent an element $\alpha_{i} \in \pi_{1}(M)$. The smallest invariant sub-group of $\pi_{1}(M)$ which contains $\alpha_{1}, \cdots, \alpha_{r}$ is independent of the orientations of $C_{1}, \cdots, C_{r}$ and of the paths joining them to $x_{0}$. We denote it by $\left\{C_{1}, \cdots, C_{r}\right\}_{M}$, or by $\left\{C_{1}, \cdots, C_{r}\right\}$ when there is no danger of confusion.

We can now state our theorem, with the usual qualifications concerning piecewise linearity (see $\$ 2$ below).

TheOREM (1.1). Let $\left(C_{1}, \cdots, C_{r}\right)$ be a set of disjoint, nonsingular 1spheres in $M$ which constitute the boundary of a Dehn surface of type $(0, r)$. Let $\left\{C_{1}, \cdots, C_{r}\right\} \subset \omega(M)$. Then a nonvacuous sub-set of $\left(C_{1}, \cdots, C_{r}\right)$, say $\left(C_{1}, \cdots, C_{q}\right)(0<q \leqq r)$, constitute the boundary of a nonsingular surface of type $(0, q)$.

If $r=1$, then $\left\{C_{1}\right\}=1 \in \omega(M)$ and (1.1) reduces to Dehn's lemma.

2. Preliminaries. It is to be understood that every $n$-manifold $(n=1,2,3)$ to which we refer has a definite piecewise affine structure 
and that all our maps are piecewise linear. Likewise a singular (or nonsingular) curve or surface in a 3-manifold will mean the image of some standard polyhedron in a piecewise linear map. As in [2] a singular surface in a 3-manifold $M$ will be called normal if it has no singularities other than double lines, triple points and branch points. It will be called canonical if it is normal and has no branch points.

By a Dehn set of curves in $M$ we shall mean a finite, nonvacuous set of nonsingular, disjoint (polyhedral) 1-spheres whose union is the boundary of a Dehn surface of genus 0 . Such a set will be called good (bad) if it contains (does not contain) a nonvacuous sub-set which constitutes the boundary of a nonsingular Dehn surface of genus 0 . Thus (1.1) states that the set $\left(C_{1}, \cdots, C_{r}\right)$ is good provided $\left\{C_{1}, \cdots, C_{r}\right\} \subset \omega(M)$. If $r=1$, then $C_{1}$ is called a Dehn curve. Any nonsingular (polyhedral) closed curve in $\dot{M}$ is obviously a Dehn curve if it is inessential in $M$.

Let $D$ be a Dehn surface in $M$. According to our conventions there is a (rectilinear) triangulation, $K$, of $M$ with a sub-complex, $L$, which covers $D$. Let $K^{\prime \prime}, L^{\prime \prime}$ be the second derived complexes of $K, L$ and let $V$ be the union of all the (closed) simplexes of $K^{\prime \prime}$ which contain vertices of $L^{\prime \prime}$ in $D-\dot{D}$. Then $V$ is a bounded 3-manifold, $\dot{D} \subset \dot{V}$ and $D$ is a deformation retract of $V$.

Let $\alpha \in \pi_{1}(V)$. Then $\alpha \in \omega(V)$ if, and only if, the transport of an indicatrix round a loop representing $\alpha$ preserves local orientation. Hence it follows that $\left\{C_{1}, \cdots, C_{r}\right\}_{r} \subset \omega(V)$ if, and only if, $\left\{C_{1}, \cdots, C_{r}\right\}_{M} \subset \omega(M)$. Therefore we may replace $M$ by $V$ when proving (1.1).

3. Proof of Dehn's lemma. Let $C$ be a Dehn curve on the boundary of a compact, connected 3-manifold $V$.

Lemma (3.1). If $V$ has no 2-sheeted cover, then $C$ is good.

Proof. Assume that $V$ has no 2-sheeted cover. Then it is orientable. If $H_{1}(V)$ has infinite order, then, since $H_{1}(V)$ is finitely generated, it has a cyclic infinite direct summand. There is, therefore, an epimorphism $H_{1}(V) \rightarrow Z_{2}$. On composing this with the Hurewicz homomorphism $\pi_{1}(V) \rightarrow H_{1}(V)$ we have an epimorphism $\phi: \pi_{1}(V) \rightarrow Z_{2}$. The kernel of $\phi$ has index 2 and determines a 2-sheeted cover of $V$, contrary to our assumption. Therefore $H_{1}(V)$ is finite and, since $V$ is orientable, every component of $\dot{V}$ is a 2-sphere [3, p. 223]. Thus $C$ lies in a nonsingular 2-sphere in $\dot{V}$ and (3.1) follows.

Let $C, V$ be as above and now assume that $V$ has a 2-sheeted cover $V_{1}$. Let $p: V_{1} \rightarrow V$ be the projection and $\tau: V_{1} \rightarrow V_{1}$ the covering trans- 
formation other than the identity. Then $p^{-1} C=C_{1} \cup_{\tau} C_{1}$, where $C_{1}$ is a Dehn curve in $\dot{V}_{1}$, and $C_{1} \cap \tau C_{1}=\phi$.

Lemma (3.2). If $C_{1}$ is good, so is $C$.

Proof. Let $C_{1}=\dot{D}_{1}$, where $D_{1}$ is a nonsingular disc. We assume, as we obviously may, that $D_{1} \cap \dot{V}_{1}=C_{1}$, whence $C_{1} \cap \tau D_{1}=\tau C_{1} \cap D_{1}=\phi$. Then $p D_{1}=D$, say, is a Dehn disc bounded by $C$. Clearly $D$ may be normalized without introducing singularities in $D_{1}$ (each step in the normalization is a "local isotopy"). So we assume that $D$ is normal, and hence canonical because $p$ is a local homeomorphism. Then $D_{1} \cap \tau D_{1}$ consists of disjoint, nonsingular closed curves and $D$ has no triple points. Since there are no triple points the lemma follows from Dehn's original argument $[1$; see also 2 , no. 14$]$.

Now let $C$ be a given Dehn curve in $M$ and $D$ a canonical Dehn disc bounded by $C$. Let $d(D)$ denote the number of closed (possibly self-interesting) double curves in $D$. Let $V$ be as in $\S 2$. Thus $D \subset V$ $\subset M, C \subset \dot{V}$ and $D$ is a deformation retract of $V$. Let $V$ have a double cover $V_{1}$ and let $p: V_{1} \rightarrow V, \tau: V_{1} \rightarrow V_{1}$ be as above. Let $D$ be canonical with respect to a map $f: \Delta \rightarrow V$, where $\Delta$ is a 2 -simplex $(D=f \Delta)$, and let $f_{1}: \Delta \rightarrow V_{1}$ be such that $p f_{1}=f$. Then $f_{1} \Delta=D_{1}$, say, is a canonical Dehn disc in $V_{1}$ and $p^{-1} D=D_{1} \cup_{\tau} D_{1}$. Since $D_{1} \cap_{\tau} D_{1} \neq \phi$, because $D$ is a deformation retract of $V$, it follows without difficulty (cf. (9.1) in [2]) that $d\left(D_{1}\right)<d(D)$. Therefore Dehn's lemma follows from (3.1), induction on $d(D)$ and (3.2).

4. Proof of (1.1). Let $C_{1}, \cdots, C_{r}(r>0)$ be a set of disjoint, nonsingular 1-spheres on the boundary of a connected, compact 3-manifold $V$. Let $C_{i}$ also denote a basic 1-cycle carried by the 1 -sphere $C_{i}$ and let $m_{1} C_{1}+\cdots+m_{r} C_{r}=\partial D$, where $m_{i}>0$ and $D$ is a singular 2-chain in $V$. This condition is obviously satisfied if $\left(C_{1}, \cdots, C_{r}\right)$ is a Dehn set. Let $\left\{C_{1}, \cdots, C_{r}\right\} \subset \omega(V)$.

LEMma 4.1. If $V$ has no 2-sheeted covering, $p: V_{1} \rightarrow V$, such that $\left\{C_{1}, \cdots, C_{r}\right) \subset p_{*} \pi_{1}\left(V_{1}\right)$, then some nonvacuous sub-set of $\left(C_{1}, \cdots, C_{r}\right)$ is a good Dehn set.

Proof. Assume that there is no such covering. Then $V$ is orientable because $\left\{C_{1}, \cdots, C_{r}\right\} \subset \omega(V)$. Let $G$ be the image of the injection $H_{1}\left(C_{1} \cup \ldots \cup C_{r}\right) \rightarrow H_{1}(V)$. The Hurewicz homomorphism $\pi_{1}(V)$ $\rightarrow H_{1}(V)$ obviously carries $\left\{C_{1}, \cdots, C_{r}\right\}$ into $G$. Therefore it follows from the first part of the proof of (3.1), with $H_{1}(V)$ replaced by $H_{1}(V) / G$, that $H_{1}(V) / G$ is a finite group. Therefore every 1-cycle in $V$ is homologous, with rational coefficients, to a linear combination 
of $C_{1}, \cdots, C_{r}$. The algebraic intersection ${ }^{1} C_{i} \cdot D$ is 0 since $V$ and hence $\dot{V}$ are orientable. Therefore it follows that there is no 1 -cycle $C_{0}$ in $\dot{V}$ such that $C_{0} \cdot C_{j}=1, C_{0} \cdot C_{k}=0$ if $j \neq k$. Also $p^{1}(V) \leqq r$, where $p^{1}(V)$ is the first Betti number of $V$. If $g(\dot{V})$ is the sum of the genera of all the components of $\dot{V}$, then $g(\dot{V}) \leqq p^{1}(V)[3$, p. 223]. Therefore $g(\dot{V})$ $\leqq r$.

Let $U_{1}, \cdots, U_{n}$ be the components of $\dot{V}-\left(C_{1} \cup \ldots \cup C_{r}\right)$ and let $S_{\lambda}=\bar{U}_{\lambda}$. Then $S_{\lambda}$ is a nonsingular surface of type $\left(p_{\lambda}, r_{\lambda}\right)$, say, and $\dot{S}_{\lambda} \subset C_{1} \cup \ldots \cup C_{r}$. If $C_{j} \subset \operatorname{Int}\left(S_{\lambda}\right)$, then nearby points on opposite sides of $C_{j}$ are joined by a path in $U_{\lambda}$. There is therefore an oriented closed curve $C_{0} \subset S_{\lambda}$ such that $C_{0} \cdot C_{j}=1, C_{0} \cdot C_{k}=0$ if $k \neq j$, which contradicts a previous conclusion. Therefore each $C_{i}$ is on the boundary of two of $S_{1}, \cdots, S_{n}$ and it follows that $r_{1}+\cdots+r_{n}=2 r$. Hence, if $\chi(X)$ denotes the Euler characteristic of a given space $X$ and if $\dot{V}$ has $m$ components, then

$$
2(m-g(\dot{V}))>\chi(\dot{V})=\sum_{\lambda} \chi\left(S_{\lambda}\right)=\sum_{\lambda}\left(2\left(1-p_{\lambda}\right)-r_{\lambda}\right)
$$

and $g(\dot{V})=m+\left(p_{1}-1\right)+\cdots+\left(p_{n}-1\right)+r$.

If $p_{\lambda}=0, r_{\lambda}=0$, then $S_{\lambda}$ is a nonsingular surface of type $\left(0, r_{\lambda}\right)$ bounded by a sub-set of $\left(C_{1}, \cdots, C_{r}\right)$. So we assume that $p_{\lambda}>0$ if $r_{\lambda}>0$. Then $r_{\lambda}=0$ if $p_{\lambda}-1<0$. In this case $S_{\lambda}$ is a component of $\dot{V}$ which does not contain any of $C_{1}, \cdots, C_{r}$. Since $C_{i} \subset \dot{V}$ it follows that $m+\left(p_{1}-1\right)+\cdots+\left(p_{n}-1\right)>0$, whence $g(\dot{V})>r$. This contradicts a previous conclusion and (4.1) is proved.

Let $\left(C_{1}, \cdots, C_{r}\right)$ be a Dehn set on $\dot{V}$ and let $p: V_{1} \rightarrow V$ be a 2sheeted covering of $V$ such that $\left\{C_{1}, \cdots, C_{r}\right\} \subset p_{*} \pi_{1}\left(V_{1}\right)$. Let $S$ be a nonsingular surface of type $(0, r)$ and $f: S \rightarrow V$ a map whose image is a Dehn surface bounded by $C_{1} \cup \ldots \cup C_{r}$. Clearly

$$
f_{*} \pi_{1}(S) \subset\left\{C_{1}, \cdots, C_{r}\right\} \subset p_{*} \pi_{1}\left(V_{1}\right) .
$$

Therefore $f$ can be lifted to a map $f_{1}: S \rightarrow V_{1}$ such that $p f_{1}=f$. Then $f_{1} S$ is a Dehn surface of type $(0, r)$ and $f_{1} \dot{S}$ is the union of a Dehn set of curves $\left(C_{1}^{\prime}, \cdots, C_{r}^{\prime}\right)$ such that $C_{i}=p C_{i}^{\prime}$. Moreover $p^{-1} C_{i}=C_{i}^{\prime}$ $\cup_{\tau} C_{i}^{\prime}, C_{i}^{\prime} \cap \tau C_{i}^{\prime}=\phi$, where $\tau: V_{1} \rightarrow V_{1}$ is the covering transformation other than the identity.

Lemma (4.2). If the set $\left(C_{1}^{\prime}, \cdots, C_{r}^{\prime}\right)$ is good, so is $\left(C_{1}, \cdots, C_{r}\right)$.

1 This refers to the intersection pairing $H_{1}(V) \times H_{2}(V, \dot{V}) \rightarrow Z$. If $i_{*}: H_{q}(\dot{V}) \rightarrow H_{q}(V)$ is the injection and $\partial: H_{2}(V, \dot{V}) \rightarrow H_{1}(\dot{V})$ the boundary homomorphism, then, $i_{*} a \cdot b= \pm i_{*}(a \cdot \partial b)$. (Cf. $[4$, pp. 171,172$]$.) 
The proofs of (4.2) and then of (1.1) are essentially the same as those of (3.2) and Dehn's lemma. The details are left to the reader.

\section{REFERENCES}

1. M. Dehn, Über die Topologie des dreidimensionalen Raumes, Math. Ann. vol. 69 (1910) pp. 137-168.

2. C. D. Papakyriakopoulos, Dehn's lemma and asphericity of knots, Ann. of Math. vol. 66 (1957) pp. 1-26.

3. H. Seifert and W. Threlfall, Lehrbuch der Topologie, Leipzig, 1934.

4. R. Thom, Espaces fibrés en sphères et carrés de Steenrod, Ann. Sci. École Norm. Sup. (3) vol. 69 (1952) pp. 109-182.

\section{BRANDEIS UNIVERSITY AND OXFORD UNIVERSITY}

\section{RESEARCH PROBLEMS}

\section{Richard Bellman: Ordinary differential equations.}

It is known that if

a. $A$ is a stability matrix, i.e., all characteristic roots have negative real parts,

b. $\|g(x)\| /\|x\| \rightarrow 0$ as $\|x\| \rightarrow 0,\left(\|x\|=\sum_{i}\left|x_{i}\right|\right)$, then all solutions of $d x / d t=A x+g(x)$ approach zero as $t \rightarrow \infty$, provided that $\|x(0)\|$ is sufficiently small (Poincaré-Lyapunov theorem).

If $x(0)=a_{1} c$, where $c$ is a characteristic vector of $A$ and $a_{1}$ is a scalar, what is the precise bound for $\left|a_{1}\right|$ in terms of $A$ and $g(x)$ ? (Received January 7, 1958.)

\section{Richard Bellman: Partial differential equations.}

It is known that if $|g(u)| /|u| \rightarrow 0$ as $u \rightarrow 0$, then the solution of $u_{t}=u_{x x}+g(u)$, $u(0, t)=u(1, t)=0, t>0$, approaches zero as $t \rightarrow \infty$, provided that $\operatorname{Max}_{0 \leqq x \leqq 1}|u(x, 0)|$ is sufficiently small.

a. If $u(x, 0)=c_{1}$ what is the precise bound for $\left|c_{1}\right|$ in terms of $g(u)$ ?

b. If $u(x, 0)=c_{1} \sin k \pi x$, what is the precise bound for $\left|c_{1}\right|$ in terms of $g(u)$ ?

\section{Richard Bellman: Functional equations.}

Let $f_{n}(u)$ be an analytic function of the function $u(x)$ and its first $n$ derivatives $u^{\prime}(x), \cdots, u^{(n)}(x)$, for $u \neq 0$, satisfying the functional equation

$$
f_{n}(u v)=f_{n}(u)+f_{n}(v) \text {. }
$$

It is well-known that $f_{0}(u)=c_{1} \log u$, and under much lighter conditions, and it is easy to show that $f_{1}(u)=c_{1} \log u+c_{2} u^{\prime} / u$.

What is the analytic form of $f_{n}$ for general $n$ ? (Received January 9, 1958.)

15. Richard Bellman: Functional equations and differential equations.

Consider the $n$th order linear differential equation

$$
\frac{d^{n} u}{d t^{n}}+a_{1}(t) \frac{d^{n-1} u}{d t^{n-1}}+\cdots+a_{n}(t) u=0
$$

